\title{
Possible Involvement of Fibrocytes in Atrial Fibrosis in Patients With Chronic Atrial Fibrillation
}

\author{
Xudong Xie, MD, PhD; Yanrong Liu, MD; Shilong Gao, MD; Bifeng Wu, BSc; \\ Xiaosheng $\mathrm{Hu}, \mathrm{MD}, \mathrm{PhD}$; Junzhu Chen, $\mathrm{MD}$
}

\begin{abstract}
Background: Chronic atrial fibrillation (AF) is characterized by a remodeling process with prominent atrial fibrosis. Fibrocytes, a bone marrow-derived population of fibroblast-like cells, have been placed at the center of a number of fibrosing conditions. The purpose of this study was to evaluate the contribution of fibrocytes to atrial fibrosis in patients with chronic AF and the possible mechanisms.

Methods and Results: We enrolled 22 consecutive valvular heart disease patients with chronic AF ( $>6$ months: CAF group) and 15 valvular heart disease patients in sinus rhythm served as controls (SR group). Left atrial tissue samples were obtained during cardiac surgery. The infiltration of fibrocytes into the atrial interstitium was observed by confocal microscopy. The number of atrial fibrocytes was approximately three-fold higher in the CAF group compared with the SR controls, and positively correlated with both the atrial collagen volume fraction $(r=0.713 ; P=0.0002)$ and the left atrial volume index $(r=0.631 ; P=0.002)$. In the peripheral blood samples collected before the operation, approximately 2.5 -fold higher percentage of circulating fibrocytes was identified in the CAF group. These fibrocytes showed a stronger proliferative capacity $(\approx 2.5$-fold $)$ and higher level expression of collagen I and $a$-SMA $(\approx 2$-fold and 4-fold, respectively) compared with the SR controls.
\end{abstract}

Conclusions: The results suggested that fibrocytes may be involved in atrial fibrosis in chronic AF through enhanced profibrotic characteristics. (Circ J 2014; 78: 338-344)

Key Words: Atrial fibrillation; Atrial remodeling; Fibrocytes; Fibrosis; Myofibroblasts

C hronic atrial fibrillation (AF) is characterized by a remodeling process that involves prominent atrial fibrosis. It has been clearly demonstrated that enhancement of atrial fibrotic remodeling provides a sufficient substrate for AF. ${ }^{1-3}$ More recently, the notion of "upstream therapy", targeting the processes involved in the development of the substrate that supports AF, has increasingly become the focus of attention. ${ }^{4-6}$ Fibrocytes, a distinct population of blood-borne cells that coexpress hematopoietic cell antigens and fibroblast products, ${ }^{78}$ have been shown to play an important role in the generation of fibrosis in different fibrotic disorders, ${ }^{9-11}$ but the relationship between fibrocytes and myocardial fibrosis has not been fully investigated.

In the present study, we examined the characteristics of fibrocytes in both atrial tissue and peripheral blood samples from valvular heart disease patients with chronic $\mathrm{AF}$, and investigated the potential contribution of fibrocytes to atrial fibrosis.

\begin{abstract}
Methods
Patients

From the thoracic surgery department, we recruited 22 consecutive valvular heart disease patients with chronic $\operatorname{AF}(>6$ months) undergoing cardiac surgery for mitral valve replacement. Patients were excluded if they had additional cardiac diseases such as coronary artery disease, New York Heart Association class III or IV congestive heart failure, or serious other systemic diseases such as hypertension, diabetes mellitus, nephropathy, connective tissue diseases, cirrhosis and pulmonary disease. The patients with administration of renin angiotensin system (RAS) blockers (ie, angiotensin-converting enzyme inhibitors or angiotensin-receptor blockers) for more than 6 months were also excluded during our enrollment period because of the known role of RAS in atrial fibrotic remodeling. As matched controls, we enrolled 15 valvular heart disease patients in sinus rhythm (SR group). All patients were investi-
\end{abstract}

Received June 19, 2013; revised manuscript received September 10, 2013; accepted October 3, 2013; released online November 7, 2013 Time for primary review: 12 days

Department of Cardiology, First Affiliated Hospital, Medical School of Zhejiang University, Hangzhou (X.X., S.G., B.W., X.H., J.C.); Department of Cardiology, First Affiliated Hospital, Nanjing Medical University, Nanjing (Y.L.), China

Mailing address: Professor Junzhu Chen, MD, Department of Cardiology, the First Affiliated Hospital, Medical School of Zhejiang University,

79 Qingchun Road, Hangzhou, Zhejiang Province 310003, PR China. E-mail: Dongdong2001x1@163.com

ISSN-1346-9843 doi:10.1253/circj.CJ-13-0776

All rights are reserved to the Japanese Circulation Society. For permissions, please e-mail: cj@j-circ.or.jp 
gated by routine cardiologic examination including X-ray, echocardiography and ECG before enrollment. Echocardiographic image acquisition and analyses were performed by experienced echocardiographers blinded to the clinical data. The values of the left atrial volume (LAV) and that volume indexed to body surface area (LAVi) were obtained according to previously described methods. ${ }^{12}$ Informed consent was given by all individuals or their guardians. The protocol of this study conformed to the ethical guidelines of the 1975 Declaration of Helsinki and was approved by the Ethics Review Committee of the hospital.

Left atrial tissue samples were obtained at the same stage of the surgical procedure during extracorporeal circulation, and were then immediately frozen in liquid nitrogen and stored at $-80^{\circ} \mathrm{C}$ until further investigation. Peripheral blood samples were obtained before the operation.

\section{Dual Immunofluorescence and Confocal Microscopy}

Atrial sections $(5 \mu \mathrm{m})$ were analyzed by dual immunofluorescence staining to determine the presence of fibrocytes using specific antibodies against CD45 and type I pro-collagen (proCol I). Atrial specimens were fixed with formaldehyde and permeabilized with $0.5 \%$ Triton X-100, then blocked with phosphate-buffered saline containing $1 \%$ bovine serum albumin and incubated with a pair of primary antibodies overnight at $4{ }^{\circ} \mathrm{C}$ [polyclonal goat antihuman pro-Col I antibody (Santa Cruz, dilution 1:50) and monoclonal mouse antihuman CD45

\begin{tabular}{|lcc|}
\hline Table. Patients' Characteristics & $\begin{array}{c}\text { SR controls } \\
(\mathbf{n}=\mathbf{1 5})\end{array}$ & $\begin{array}{c}\text { CAF patients } \\
(\mathbf{n = 2 2})\end{array}$ \\
Age (years) & $53 \pm 3$ & $57 \pm 2$ \\
Sex, male/female (n) & $9 / 6$ & $13 / 9$ \\
History of AF (months) & - & $26 \pm 4^{*}$ \\
LVEF Mean (\%) & $60 \pm 2$ & $55 \pm 1$ \\
LAV/BSA (ml/m²) & $25.40 \pm 11.39$ & $41.99 \pm 17.08^{\star *}$ \\
Medication & & \\
Digitalis (n) & 2 & 9 \\
Calcium antagonist $(\mathrm{n})$ & 3 & 7 \\
$\beta$-blocker $(\mathrm{n})$ & 3 & 8 \\
Class Ic/lll antiarrhythmic drug (n) & - & 4 \\
\hline
\end{tabular}

${ }^{*} \mathrm{P}<0.05,{ }^{*} \mathrm{P}<0.01$ compared with $\mathrm{SR}$ controls.

$\mathrm{AF}$, atrial fibrillation; $\mathrm{BSA}$, body surface area; $\mathrm{CAF}$, chronic atrial fibrillation; LAV, left atrial volume; LVEF, left ventricular ejection fraction; SR, sinus rhythm.

antibody (Dako, dilution 1:100)]. After thorough washing, the specimens were incubated for $1 \mathrm{~h}$ at room temperature with Alexa Fluor 488- and Alexa Fluor 546-labeled second antibodies (Molecular Probes, both 1:100). This was followed by staining with 4',6-diamidino-2-phenylindole (DAPI; Sigma, 1:10000) for $20 \mathrm{~min}$. Negative control experiments were per-
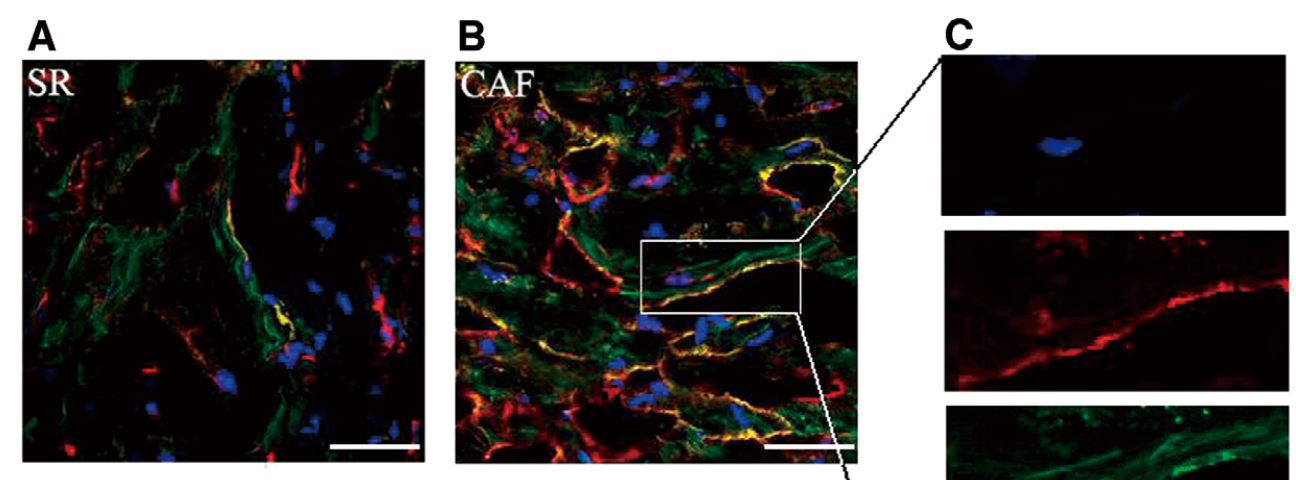

D
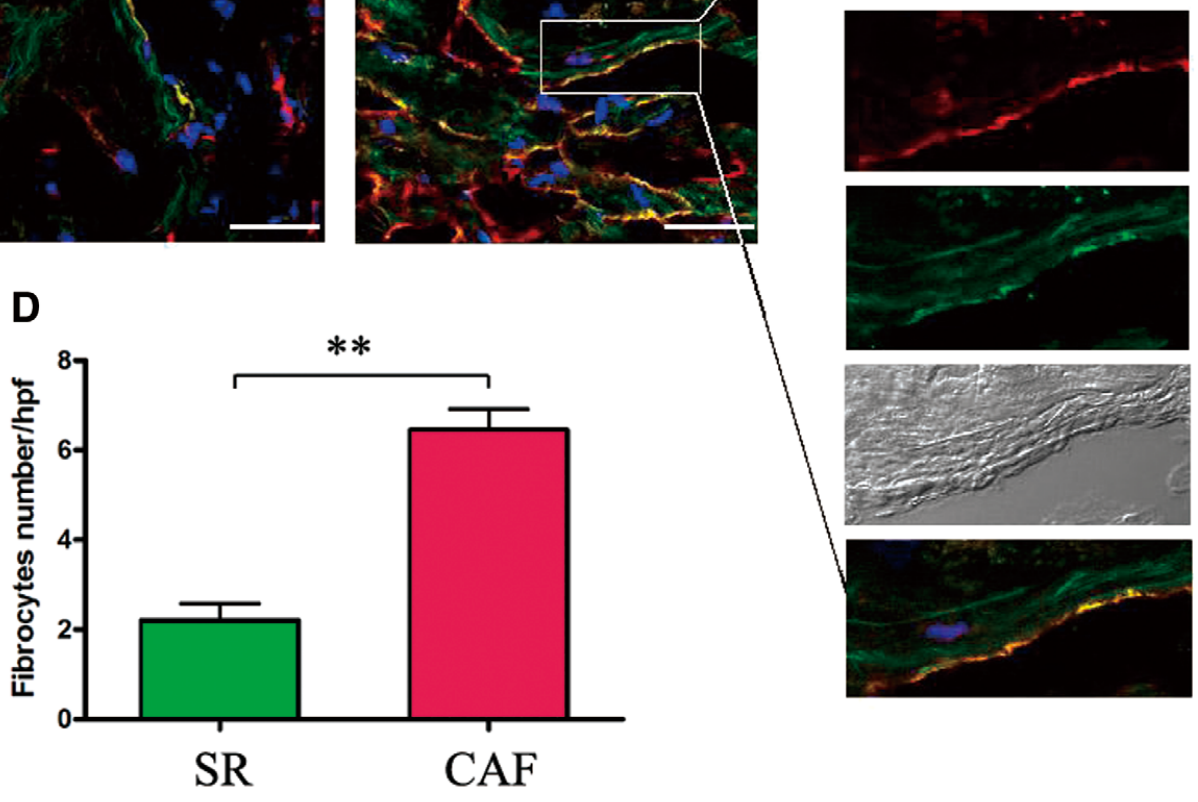

Figure 1. Fluorescence microscopy images of atrial tissue using CD45/pro-Col I for fibrocyte identification. (A,B) Representative photomicrographs from controls in sinus rhythm ( $S R, n=15)$ and chronic AF patients (CAF, $n=22)$. (C) Separate markers for CD45 (red), pro-Col I (green) and nuclei staining (blue), and a merged image (yellow) are shown. (D) Mean value and standard error of fibrocyte number per high-power field (hpf) in each group. Values are the mean $\pm \mathrm{SEM}$. ${ }^{\star *} \mathrm{P}<0.01 \mathrm{vs}$. SR group. Original magnification $\times 400(A, B)$ and $\times 800(C)$. Scale bar $=50 \mu \mathrm{m}$. Col 1, type I collagen. 
A a

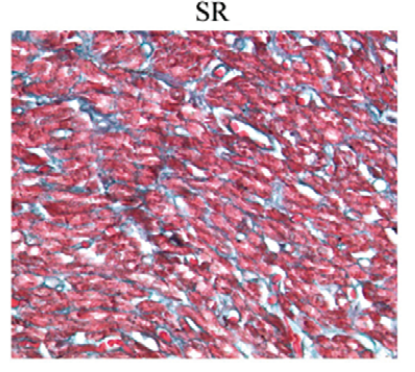

b

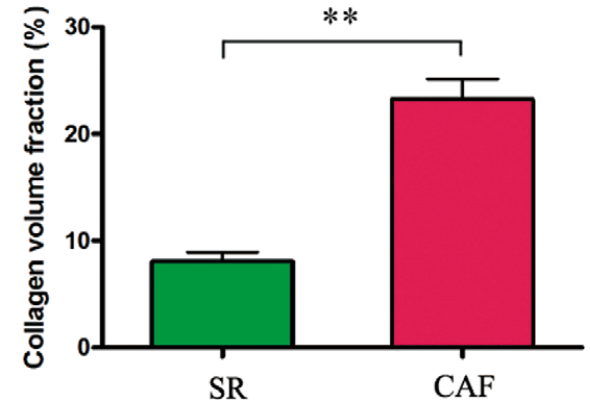

B a

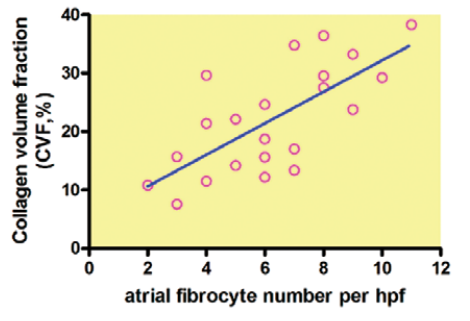

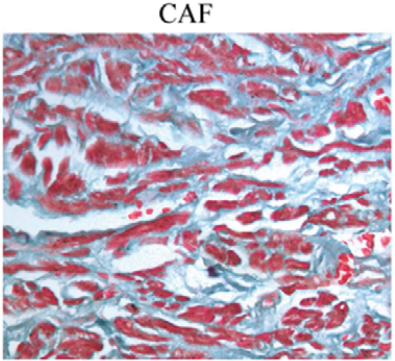

b

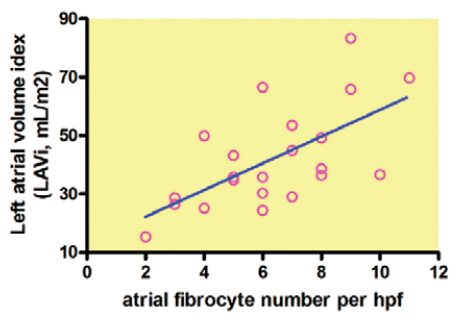

Figure 2. (A-a) Representative examples of Masson's trichrome staining of atrial biopsy specimens from the sinus rhythm controls (SR, $n=15)$ and chronic AF patients (CAF, $n=22)$. Collagen stains blue (×200). (A-b) Quantitative analysis of the collagen volume fraction in the SR and CAF groups. Data are mean \pm SEM. ${ }^{* *} \mathrm{P}<0.01$ vs. SR group. (B-a) Association between the number of $\mathrm{CD} 45^{+} /$pro-Col $\mathrm{I}^{+}$atrial fibrocytes per high-power field (hpf) and collagen volume fraction (CVF) in chronic AF patients ( $r=0.713 ; P=0.0002)$. (B-b) Association between the number of CD45+/pro-Col I+ atrial fibrocytes per hpf and left atrial volume index (LAVi, $\mathrm{ml} / \mathrm{m}^{2}$ ) measured by echocardiography in chronic AF patients $(r=0.631 ; P=0.002)$. Col 1 , type I collagen. formed by replacing the primary antibody with serum, or sections were incubated with each primary antibody followed by inappropriate secondary antibody to determine that each secondary antibody was specific to the species against which it was made. Confocal microscopy was performed using a fluorescence laser scanning confocal microscope (Olympus Fluoview FV 1000). Mean numbers of interstitial CD45/pro-Col I doublepositive fibrocytes were counted from more than 10 randomly chosen fields under high-power magnification $(\times 400)$. Two independent observers examined the immunofluorescent staining without prior knowledge of the patients' clinical courses.

\section{Culture and Characterization of Circulating Fibrocytes}

Circulating fibrocytes were isolated according to previously published methods. ${ }^{7,13}$ Briefly, peripheral blood mononuclear cells (PBMCs) were separated from whole blood by Ficoll density gradient centrifugation (Invitrogen, Carlsbad, CA, USA) following the manufacturer's protocol. Mononuclear cells at the interface were harvested, washed twice, and resuspended in Dulbecco's modified Eagle's medium containing 20\% fetal calf serum for 14 days. After 7 days of culture, non-adherent cells were removed by gentle aspiration and the media were replaced. Subsequently, the adherent cells were harvested by Accutase (Innovative Cell Tech, USA). Immunomagnetic selection was used to deplete T lymphocytes (Dynabeads antiCD2; Invitrogen) and monocytes/macrophages (Dynabeads anti-CD14; Invitrogen). The remaining fibrocytes were recul- tured for another 7 days. The purity and characteristics of the cultured fibrocyte populations were assessed by immunofluorescence and flow cytometry.

\section{Flow Cytometry}

Freshly isolated PBMCs or cultured fibrocytes $\left(1 \times 10^{6}\right.$ cells $)$ were immersed in permeabilizing solution and incubated with monoclonal mouse antihuman CD45 antibody (Dako), and polyclonal goat antihuman pro-Col I (Santa Cruz) antibody indirectly labeled with Alexa Fluor 488 or 546 as required. Expression of $\alpha$-smooth-muscle actin ( $\alpha$-SMA) were evaluated by flow cytometry using mouse monoclonal anti- $\alpha$-SMA antibody (1:50; Dako) indirectly labeled with fluorescein isothiocyanate. In addition, the proportions of PBMCs stained with CD68 (mouse antihuman CD68; 1:100; Dako) or with single pro-Col I were also detected by flow cytometry. All experiments included isotype controls. Cells $\left(2 \times 10^{5}\right)$ were flow cytometrically analyzed using CellQuest software (Becton Dickinson) and expressed as the results.

\section{Western Blotting}

The extracted cell protein of each sample ( $50 \mu \mathrm{g}$ total protein) was fractionated by $7 \%$ sodium dodecylsulfate (SDS) polyacrylamide gel electrophoresis and transblotted onto nitrocellulose filters. The membranes were incubated overnight at $4^{\circ} \mathrm{C}$ using rabbit antihuman type I collagen (Col I) (1:100 dilution; Boshide, Wuhan, China) and monoclonal anti-glyceraldehyde- 

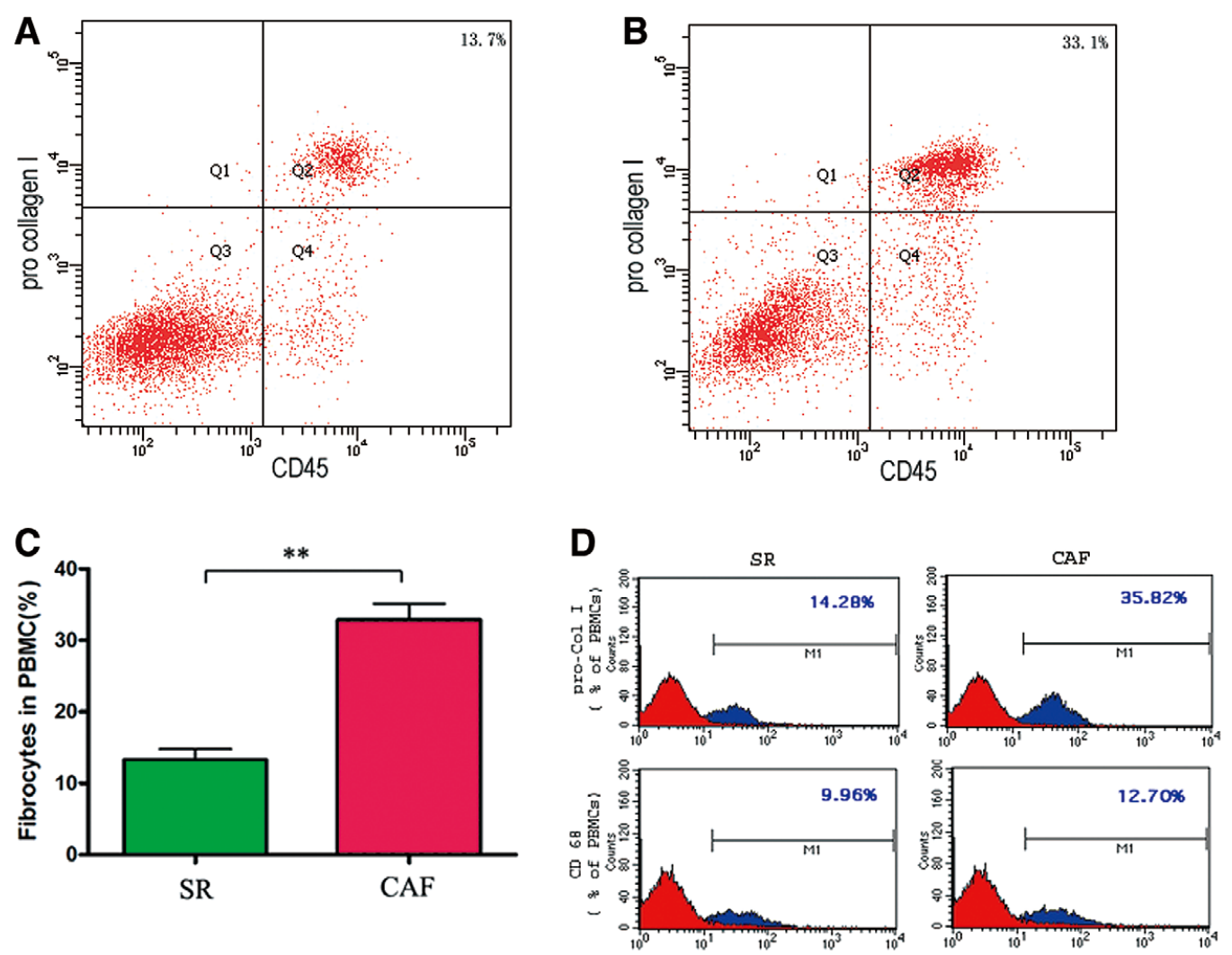

Figure 3. Flow cytometric analysis of fibrocytes among peripheral blood mononuclear cells (PBMCs) by immunostaining of CD45 and pro-Col I. (A,B) Representative dot plots of CD45+/pro-Col I+ cells from the sinus rhythm controls $(S R, n=15)$ and chronic AF patients (CAF, $n=22)$. (C) Percentage of CD45+/pro-Col I+ cells in the SR and CAF groups. Data are mean $\pm S E M$. ${ }^{* \star} P<0.01$ vs. SR group. (D) Example histograms of PBMCs stained with pro-Col I or CD68 in the SR and CAF groups with corresponding isotype controls (red histogram). Col 1, type I collagen.

3-phosphate dehydrogenase (1:1000 dilution; Chemicon International) antibodies. The membranes were then incubated for $1 \mathrm{~h}$ in the appropriate diluted secondary antibody. Signal was detected using an electrochemiluminescence kit (GE Healthcare, Little Chalfont, UK). Exposed films were quantified on an automated imaging system (Eastman Kodak ${ }^{\mathrm{TM}}$, Rochester, NY, USA). A molecular weight marker (Invitrogen) was used to estimate the molecular weight of Col I.

\section{Quantitative Morphometry of Biopsy Samples}

The atrial tissue samples were immediately fixed in $10 \%$ buffered formalin and embedded in paraffin. Tissue sections were stained with Masson trichrome stain for histological evaluation and images were acquired with a projection microscope $(\times 200)$. Subsequent image analysis was performed using HMIAS-2000 image analyzing system to determine the extent of myocardial interstitial fibrosis, which was expressed as collagen volume fraction (CVF, \%). Fibrosis appeared as blue (collagen stain) with Masson trichrome staining. The CVF was calculated as the sum of all connective tissue areas divided by the sum of all connective tissue and muscle areas averaged over 6 representative fields of the section, where there was neither endocardium nor blood vessels. This histological evaluation was performed by a well-trained pathologist without knowledge of which patient provided the tissue sections.

\section{Statistical Analysis}

Data are expressed as the mean \pm SEM. Categorical variables were compared using the chi-square or Fisher's exact test. Continuous variables were assessed by independent-samples Student's t-test or one-way analysis of variance (ANOVA) using SPSS 18 software (Chicago, IL, USA). For correlation analysis, Pearson's correlation was calculated. $\mathrm{P}<0.05$ was considered significant.

\section{Results}

\section{Patients' Characteristics}

Clinical characteristics of the patient population are summarized in Table. There were no significant differences in age, sex or left ventricular ejection fraction between the 2 groups. LAVi was higher in chronic AF patients than in those in the SR group $(\mathrm{P}<0.01)$.

\section{Number and Site of Fibrocytes in Patients With Chronic AF}

The presence of fibrocytes in atrial tissues was determined by confocal microscopy. One of the unique characteristics of fibrocytes is their simultaneous expression of both CD45 and type I collagen. We found that $\mathrm{CD}^{+} 5^{+} / \mathrm{pro}-\mathrm{Col} \mathrm{\textrm {I } ^ { + }}$ fibrocytes had infiltrated the atrial interstitium (Figure 1). More doublepositive fibrocytes were detected in the CAF group compared with the SR group (6.1 \pm 0.5 vs. $2.2 \pm 0.4$ per high-power field, 
A
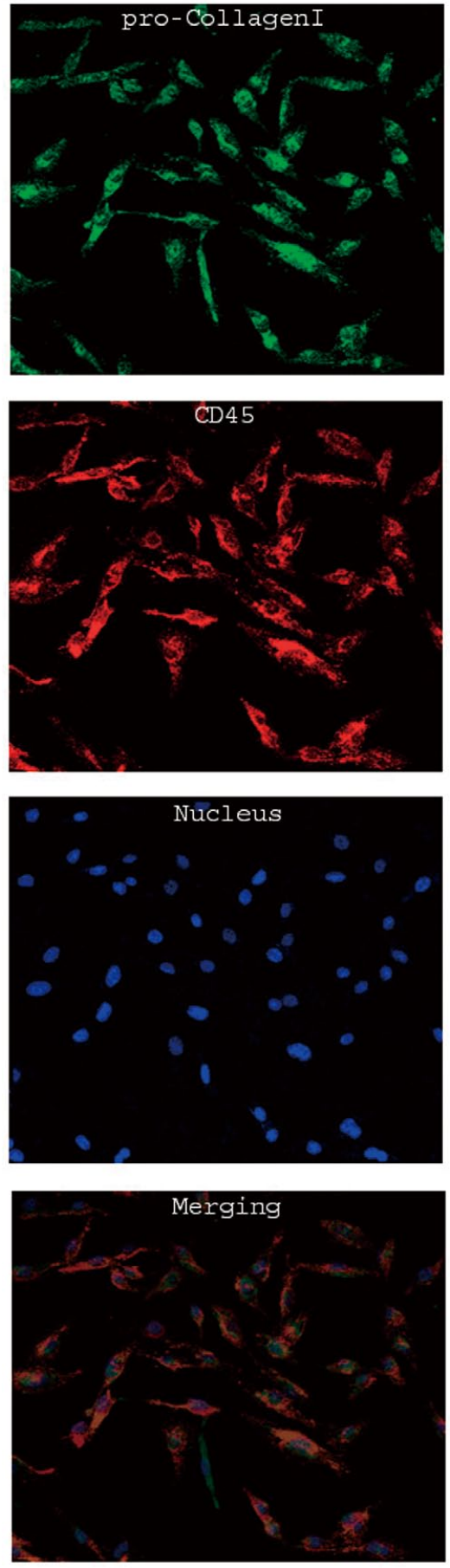

B

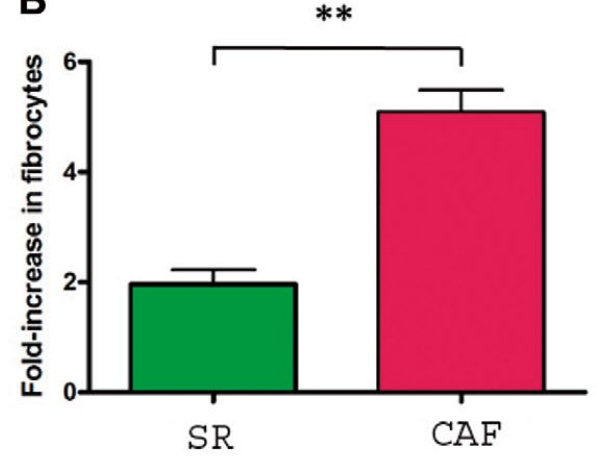

C
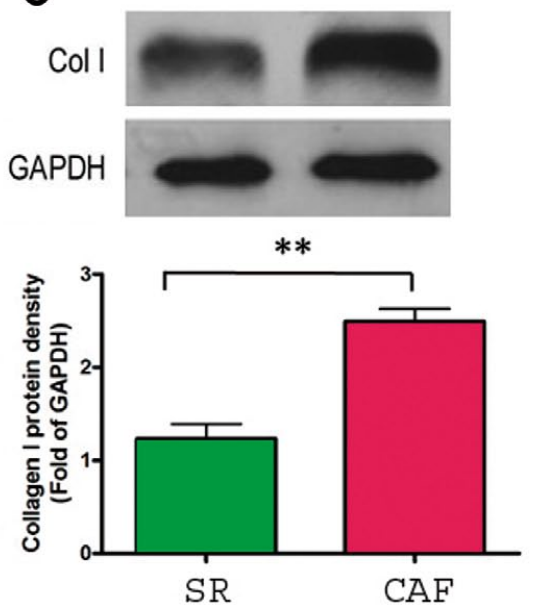

D

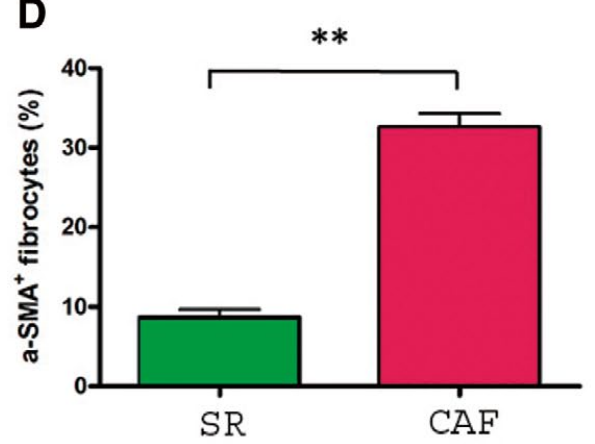

Figure 4. (A) Representative photomicrographs of cultured fibrocyte morphology. Fibrocytes were identified by dual immunofluorescence staining using CD45 and pro-Col I. The separate markers for CD45 (red), pro-Col I (green) and nuclei staining (blue), and a merged image (yellow) are shown (magnification, $\times 200$ ). (B) Proliferative capacity of fibrocytes, expressed as the ratio of fibrocytes grown after culture for 14 days compared with fibrocytes among mononuclear cells at the start of culture. (C) Expression of collagen I in circulating fibrocytes measured by western blotting. Glyceraldehyde-3-phosphate dehydrogenase (GADPH) was used as a housekeeping gene. (D) Percentage of alpha smooth muscle actin (a-SMA) -positive circulating fibrocytes determined by flow cytometric analysis. SR, sinus rhythm controls $(n=15)$; CAF, chronic AF patients $(n=22)$. Data are mean $\pm S E M$. ${ }^{* \star} P<0.01$ vs. SR group. Col 1, type I collagen. 


\section{$\mathrm{P}<0.01)$.}

As an index of fibrosis, the CVF of the atrial myocardium was nearly 3-fold higher in CAF patients than in the SR controls $(22.1 \pm 2.0 \%$ vs. $7.6 \pm 0.7 \%, \mathrm{P}<0.01)$ (Figure 2A). Given our observation of significantly elevated atrial fibrocyte accumulation in the CAF group, we sought to ascertain whether the number of atrial interstitial fibrocytes correlated with the atrial myocardial CVF. A positive correlation was found ( $\mathrm{r}=0.713$; $\mathrm{P}=0.0002$ ) in the CAF patients (Figure 2B-a). A significant correlation also existed between the number of atrial fibrocytes and LAVi ( $\mathrm{r}=0.631 ; \mathrm{P}=0.002$ ) (Figure 2B-b).

\section{Phenotype of Circulating Fibrocytes in Chronic AF Patients}

Using flow cytometry, we identified a double-positive population of $\mathrm{CD} 45^{+} /$pro-Col $\mathrm{I}^{+}$fibrocytes in the peripheral blood samples from all subjects. There was an obvious increase in the number of $\mathrm{CD} 45^{+} / \mathrm{pro}-\mathrm{Col} \mathrm{I}^{+}$cells in the CAF group vs. the SR controls $(33.7 \pm 3.0 \%$ vs. $13.2 \pm 1.8 \%, \mathrm{P}<0.01)$ (Figures $3 \mathrm{~A}-\mathrm{C}$ ). These data indicated that PBMCs from patients with chronic AF had a greater propensity for fibrocyte production. In addition, the gene expression of pro-Col I in PBMCs was increased in the CAF patients compared with the SR group, showing an almost similar pattern as observed for the number of peripheral blood fibrocytes (Figure 3D, data not shown). We also evaluated the proportion of macrophages among the PBMCs and found that the CD68 expression did not differ significantly between the CAF and SR groups (Figure 3D, data not shown).

To measure whether fibrocytes with an altered profibrotic phenotype existed in the CAF subjects, we compared the characteristics of cell proliferation, Col I production and differentiation into myofibroblasts in fibrocytes cultured from the peripheral blood samples (Figure 4). Cultured fibrocytes gradually displayed an adherent, spindle-shaped morphology with a circular or oval nucleus and were confirmed by coexpression of CD45 and pro-Col I (Figure 4A). Purity was routinely $>90 \%$ by either immunofluorescence or flow cytometry. The proliferative capacity of circulating fibrocytes was expressed as the ratio of fibrocytes grown after culture for 14 days compared with fibrocytes among mononuclear cells at the start of culture. There was a greater increase in fibrocyte number after 14 days in the CAF group compared with the SR controls (5.1 \pm 0.4 -fold increase vs. 2.0 \pm 0.3 -fold increase, $\mathrm{P}<0.01)$. The expressions of Col I and $\alpha$-SMA in circulating fibrocytes were approximately 2 -fold $(2.49 \pm 0.14$ fold vs. $1.23 \pm 0.16$ fold, $\mathrm{P}<0.01)$ and 4 -fold $(32.6 \pm 1.7 \%$ vs. $8.7 \pm 1.0 \%, \mathrm{P}<0.01)$ higher, respectively, in the CAF group compared with the SR controls.

\section{Discussion}

$\mathrm{AF}$ is the most commonly acquired arrhythmia in adults and substantially increases the risk of stroke and premature death. There is strong evidence to demonstrate that atrial fibrosis plays a key role in the development and persistence of $\mathrm{AF}, 1-3,14,15$ but it is unclear whether there is direct cause and effect relationship or whether these events occur as a consequence of independent pathologic changes in the heart. To the best of our knowledge, the present study describes for the first time the possible contribution of bone marrow-derived fibrocytes to atrial fibrosis in chronic AF patients who have valvular heart disease.

In the present study, we observed that cells expressing fibrocyte markers were present in the atrial interstitium and the number of these infiltrated cells increased in patients with chronic AF, which positively correlated with atrial remodeling. Our results also indicated that the circulating fibrocytes in chronic AF patients showed a higher proliferative capacity and overexpression of $\alpha$-SMA and Col I. The myofibroblasts ( $\alpha$-SMA-positive cells) are key mesenchymal cells implicated in extracellular matrix synthesis. ${ }^{16,17}$ Besides their involvement in structural remodeling, myofibroblasts have been recently discovered in vitro to promote arrhythmogenesis by direct modification of cardiomyocyte electrophysiology following establishment of heterocellular electrical coupling. ${ }^{18}$ Moreover, it has been shown that $\alpha$-SMA-containing stress fibers might be a potential target of antiarrhythmic therapy in hearts undergoing structural remodeling. ${ }^{19}$ These results support the hypothesis that fibrocytes may promote the process of atrial fibrosis by enhanced recruitment into the fibrillating atria and subsequent a great degree of differentiability into $\alpha$-SMA ${ }^{+}$ myofibroblasts. However, the precise pathogenic mechanisms remain to be determined.

It is known that injury to the heart causes hematopoietic progenitor cells to migrate to the site of damage and to undergo progenitor cell differentiation. ${ }^{20-22}$ Interestingly, Goette et al demonstrated a high proportion of $\mathrm{CD} 34^{+}$hematopoietic progenitor cells in the blood of patients with persistent AF, and these had a greater tendency to differentiate into cells expressing myocyte markers. ${ }^{23}$ There are other reports that suggest that bone marrow-derived cells might be involved in the pathogenesis of AF, but the exact cell type(s) still remain unclear. ${ }^{24,25}$ Based on our results, we hypothesize that AF might trigger the distinct fibrotic response of fibrocytes with enhanced differentiation into fibroblasts/myofibroblasts and subsequently worsen the pathogenesis of atrial fibrosis. It is possible that a cyclical relationship between $\mathrm{AF}$ and atrial fibrosis may lead to degenerative atrial changes with increased episodes of $\mathrm{AF}$ and even $\mathrm{AF}$ persistence, giving rise to the concept that "AF begets AF" ${ }^{26,27}$ More studies are needed to investigate the regulation of chemokine gene expression during the activation of fibrocytes, which will highlight the potential of antifibrotic drugs to successfully treat AF.

The present data shed light on the possible involvement of fibrocytes in atrial fibrosis in AF patients by regulating the number and activation of fibrocytes. Further investigations are needed to elucidate the precise relationships among fibrocytes, atrial fibrogenesis, and the development of AF.

\section{Acknowledgments}

This work was supported by funds from the National Natural Science Foundation of China (30900614, 81270002, 81070082 and 81170167).

\section{Disclosures}

The authors declare no conflicts of interest and no financial support from any commercial entity.

\section{References}

1. Tan AY, Zimetbaum P. Atrial fibrillation and atrial fibrosis. J Cardiovasc Pharmacol 2011; 57: 625-629.

2. Burstein B, Nattel S. Atrial fibrosis: Mechanisms and clinical relevance in atrial fibrillation. J Am Coll Cardiol 2008; 51: 802-809.

3. Kiryu M, Niwano S, Niwano H, Kishihara J, Aoyama Y, Fukaya H, et al. Angiotensin II-mediated up-regulation of connective tissue growth factor promotes atrial tissue fibrosis in the canine atrial fibrillation model. Europace 2012; 14: 1206-1214.

4. Takahashi N, Kume O, Wakisaka O, Fukunaga N, Teshima Y, Hara $\mathrm{M}$, et al. Novel strategy to prevent atrial fibrosis and fibrillation. Circ J 2012; 76: 2318-2326.

5. Burstein B, Nattel S. Atrial structural remodeling as an antiarrhythmic target. J Cardiovasc Pharmacol 2008; 52: 4-10.

6. Takahashi N, Kume O, Wakisaka O, Fukunaga N, Teshima Y, Hara $\mathrm{M}$, et al. Novel strategy to prevent atrial fibrosis and fibrillation. Circ J 2012; 76: 2318-2326. 
7. Bucala R, Spiegel LA, Chesney J, Hogan M, Cerami A. Circulating fibrocytes define a new eukocyte subpopulation that mediates tissue repair. Mol Med 1994; 1: 71-81.

8. Curnow SJ, Fairclough M, Schmutz C, Kissane S, Denniston AK, Nash K, et al. Distinct types of fibrocyte can differentiate from mononuclear cells in the presence and absence of serum. PLoS One 2010; 5: e9730.

9. Herzog EL, Bucala R. Fibrocytes in health and disease. Exp Hematol 2010; 38: $548-556$

10. Quan TE, Cowper SE, Bucala R. The role of circulating fibrocytes in fibrosis. Curr Rheumatol Rep 2006; 8: 145-150.

11. Reich B, Schmidbauer K, Rodriguez Gomez M, Johannes Hermann F, Göbel N, Brühl H, et al. Fibrocytes develop outside the kidney but contribute to renal fibrosis in a mouse model. Kidney Int 2013; 84: $78-89$.

12. Kim YH, Lee SC, Her AY, Kim HJ, Choi JO, Shin DH, et al. Preoperative left atrial volume index is a predictor of successful sinus rhythm restoration and maintenance after the maze operation. $J$ Thorac Cardiovasc Surg 2007; 134: 448-453.

13. Saunders R, Siddiqui S, Kaur D, Doe C, Sutcliffe A, Hollins F, et al. Fibrocyte localization to the airway smooth muscle is a feature of asthma. J Allergy Clin Immunol 2009; 123: 376-384.

14. Ko WC, Hong CY, Hou SM, Lin CH, Ong ET, Lee CF, et al. Elevated expression of connective tissue growth factor in human atrial fibrillation and angiotensin II-treated cardiomyocytes. Circ J 2011; 75: $1592-1600$

15. Choi EK, Chang PC, Lee YS, Lin SF, Zhu W, Maruyama M, et al. Triggered firing and atrial fibrillation in transgenic mice with selective atrial fibrosis induced by overexpression of TGF- $\beta 1$. Circ J 2012; 76: $1354-1362$.

16. Scotton CJ, Chambers RC. Molecular targets in pulmonary fibrosis: The myofibroblast in focus. Chest 2007; 132: 1311-1321.

17. Liew R, Khairunnisa K, Gu Y, Tee N, Yin NO, Naylynn TM, et al Role of tumor necrosis factor- $\alpha$ in the pathogenesis of atrial fibrosis and development of an arrhythmogenic substrate. Circ J 2013; 77:
$1171-1179$.

18. Rohr S. Myofibroblasts in diseased hearts: New players in cardiac arrhythmias? Heart Rhythm 2009; 6: 848-856.

19. Zhang YM, Hartzell C, Narlow M, Dudley SC Jr. Stem cell-derived cardiomyocytes demonstrate arrhythmic potential. Circulation 2002; 106: $1294-1299$.

20. Kocher AA, Schuster MD, Bonaros N, Lietz K, Xiang G, Martens $\mathrm{TP}$, et al. Myocardial homing and neovascularization by human bone marrow angioblasts is regulated by IL-8/Gro CXC chemokines. $J$ Mol Cell Cardiol 2006; 40: 455-464.

21. Kocher AA, Schuster MD, Szabolcs MJ, Takuma S, Burkhoff D, Wang J, et al. Neovascularization of ischemic myocardium by human bone-marrow-derived angioblasts prevents cardiomyocyte apoptosis, reduces remodeling and improves cardiac function. Nat Med 2001; 7: 430-436.

22. Shintani S, Murohara T, Ikeda H, Ueno T, Honma T, Katoh A, et al. Mobilization of endothelial progenitor cells in patients with acute myocardial infarction. Circulation 2001; 103: 2776-2779.

23. Goette A, Jentsch-Ullrich K, Lendeckel U, Röcken C, Agbaria M, Auricchio A, et al. Effect of atrial fibrillation on hematopoietic progenitor cells: A novel pathophysiological role of the atrial natriuretic peptide? Circulation 2003; 108: 2446-2449.

24. Hu YF, Yeh HI, Tsao HM, Tai CT, Lin YJ, Chang SL, et al. Impact of circulating monocyte CD36 level on atrial fibrillation and subsequent catheter ablation. Heart Rhythm 2011; 8: 650-656.

25. Liu L, Lee J, Fu G, Liu X, Wang H, Zhang Z, et al. Activation of peripheral blood CD3(+) T-lymphocytes in patients with atrial fibrillation. Int Heart J 2012; 53: 221 - 224.

26. Maurits CEF, Wijffels MD, Charles JHJ, Dorland R, Allessie MA. Atrial fibrillation begets atrial fibrillation: A study in awake chronically instrumented goats. Circulation 1995; 92: 1954-1968.

27. Pellman J, Lyon RC, Sheikh F. Extracellular matrix remodeling in atrial fibrosis: Mechanisms and implications in atrial fibrillation. $J$ Mol Cell Cardiol 2010; 48: 461-467. 